\title{
Acute Basilar Artery Occlusion: Differences in Characteristics and Outcomes after Endovascular Therapy between Patients with and without Underlying Severe Atherosclerotic Stenosis
}

\author{
(D)Y.Y. Lee, (D)W. Yoon, (1) S.K. Kim, DB.H. Baek, (D) G.S. Kim, DJ.T. Kim, and (D).S. Park
}

\begin{abstract}
BACKGROUND AND PURPOSE: Prediction of underlying intracranial atherosclerotic stenosis before endovascular therapy might be helpful for appropriate therapeutic planning in patients with acute ischemic stroke. This study aimed to compare the characteristics and treatment outcomes in patients with acute basilar artery occlusion relative to the existence or nonexistence of underlying intracranial atherosclerotic stenosis.
\end{abstract}

MATERIALS AND METHODS: Sixty-two patients with acute basilar artery occlusion underwent multimodal endovascular therapy. All patients underwent stent-retriever thrombectomy as a first-line endovascular therapy. Patients with underlying intracranial atherosclerotic stenosis underwent additional intracranial angioplasty and stent placement. The clinical and imaging characteristics and treatment outcomes were retrospectively analyzed and compared between patients with and without intracranial atherosclerotic stenosis.

RESULTS: Underlying intracranial atherosclerotic stenosis was identified at the occlusion site in 15 patients (24.1\%). Occlusion in the proximal segment of the basilar artery was more common in patients with intracranial atherosclerotic stenosis $(60 \%$ versus $6.4 \%, P<.001)$, whereas occlusion in the distal segment was more common in those without it ( $91.5 \%$ versus $26.7 \%, P<.001)$. Bilateral thalamic infarction on a pretreatment DWI was less common in patients with intracranial atherosclerotic stenosis $(0 \%$ versus $27.7 \%, P=.027)$ compared with those without it. There were no significant differences in the rates of successful revascularization, favorable outcome, symptomatic hemorrhage, and mortality between the 2 groups.

CONCLUSIONS: Underlying intracranial atherosclerotic stenosis was not uncommon in patients with acute basilar artery occlusion. The occlusion segment of the basilar artery and the presence or absence of bilateral thalamic infarction on a pretreatment DWI might be helpful for predicting underlying intracranial atherosclerotic stenosis in patients with acute basilar artery occlusion. Patients with and without underlying intracranial atherosclerotic stenosis who underwent endovascular therapy had similar outcomes.

ABBREVIATIONS: BAO = basilar artery occlusion; ICAS = intracranial atherosclerotic stenosis

A cute ischemic stroke due to basilar artery occlusion (BAO) is associated with the highest mortality and morbidity among strokes attributable to intracranial large-vessel occlusions. ${ }^{1,2}$ Morbidity and mortality rates have been reported to be up to $95 \%$, and the chances for good outcome are extremely low without active treatment. ${ }^{2,3}$ The rates of death or dependency have been $76 \%-78 \%$, even when patients with acute BAO were treated with intravenous or intra-arterial thrombolysis. ${ }^{3}$ Stent-retriever

Received February 9, 2017; accepted after revision March 24.

From the Departments of Radiology (Y.Y.L., W.Y., S.K.K., B.H.B., G.S.K.) and Neurology (J.T.K., M.S.P.), Chonnam National University Medical School, Chonnam National University Hospital, Gwangju, Republic of Korea.

Please address correspondence to Woong Yoon, MD, Department of Radiology, Chonnam National University Hospital, 42 Jebong-ro, Dong-gu, Gwangju, 61469, Republic of Korea; e-mail: radyoon@jnu.ac.kr

http://dx.doi.org/10.3174/ajnr.A5233 thrombectomy is increasingly being used in patients with acute $\mathrm{BAO}$ and shows a high recanalization rate. ${ }^{4}$ Many case series have shown that stent-retriever thrombectomy is a safe and feasible treatment technique in patients with acute BAO. ${ }^{4-7}$

Embolism from cardiac or proximal large-artery sources and in situ thrombosis superimposed on underlying intracranial atherosclerotic stenosis (ICAS) have been identified as major causes of acute BAO. ${ }^{8}$ ICAS is one of the most common causes of ischemic stroke worldwide and is particularly prevalent in Asian, black, Hispanic, and Indian populations. ${ }^{9}$ Strategies for endovascular therapy in patients with acute stroke with underlying ICAS should be different from those without it. In this regard, prediction of underlying ICAS before endovascular therapy might be important and helpful to neurointerventionalists in the planning of endovascular therapy in patients with acute BAO; however, this has not yet been systematically studied, to our knowledge. In ad- 
dition, very few studies have investigated outcomes after endovascular therapy in patients with acute BAO due to underlying ICAS. ${ }^{10,11}$ Accordingly, this study aimed to investigate the imaging and clinical characteristics for predicting underlying ICAS in patients with acute BAO and to compare the clinical outcomes of endovascular therapy in those patients relative to the existence or nonexistence of underlying ICAS.

\section{MATERIALS AND METHODS \\ Patients}

From December 2010 to February 2016, 62 patients with acute stroke due to BAO received stent-retriever thrombectomy at a regional comprehensive stroke center. We retrospectively analyzed clinical and radiologic data of these 62 patients, including age, sex, stroke risk factors, intravenous thrombolysis, time to procedure, procedural time, time to revascularization, and etiology of stroke according to the Trial of Org 10172 in Acute Stroke Treatment (TOAST) classification. ${ }^{12}$

On admission, all patients were assessed with the NIHSS by a stroke neurologist. Patients underwent nonenhanced CT and MR imaging before endovascular therapy. The inclusion criteria for stent-retriever thrombectomy were as follows: 1) presentation within 12 hours of stroke onset, 2) baseline NIHSS score of $\geq 4,3$ ) no intracranial hemorrhage detected on cranial CT or MR imaging, 4) BAO detected with MR angiography and conventional angiography, 5) no bilateral diffuse pontine ischemia on DWI, and 6) a premorbid mRS score of $\leq 2$. The institutional ethics committee approved this retrospective analysis and waived the requirements for informed consent on the basis of study design.

\section{Endovascular Therapy}

All endovascular therapy was performed by one interventional neuroradiologist with 14 years of experience in neurovascular intervention. Written informed consent for endovascular therapy was obtained from a family member for all patients. Cerebral angiography and endovascular therapy were performed with the patient under conscious sedation. In cases of agitation, an IV bolus of midazolam was given and repeated if necessary. After detecting arterial occlusion on diagnostic angiography, we used stent-retriever thrombectomy with a Solitaire stent (Covidien, Irvine, California) or Trevo stent (Stryker, Kalamazoo, Michigan) as the first-line endovascular therapy method. When stentretriever thrombectomy was unsuccessful, manual aspiration thrombectomy with intermediate catheters was performed as a rescue treatment. The details of the techniques used for stentretriever thrombectomy and manual aspiration thrombectomy were described previously. ${ }^{6,13}$

Underlying ICAS was determined when severe ( $\geq 70 \%$ ) stenosis of the basilar artery was seen on the initial diagnostic angiography or on the follow-up angiography after a mechanical thrombectomy procedure. ${ }^{14}$ The degree of arterial stenosis was determined with the Warfarin Aspirin Symptomatic Intracranial Disease criteria. ${ }^{15}$ If underlying ICAS was revealed, intracranial angioplasty was performed with a Gateway PTA balloon catheter (Stryker). The diameter of the balloon was undersized to $80 \%$ of the normal vessel diameter just distal to the stenosis. The balloon was inflated slowly 1 or 2 times with a screw-type pressure inflation device at
4-6 atm for 60 seconds. After balloon angioplasty, intracranial stent placement with the Wingspan stent system (Stryker) was performed whenever possible. The stent diameter was sized to exceed the diameter of the normal vessel by $0.5 \mathrm{~mm}$. The stent length was selected to cover the entire stenotic segment. All patients who underwent intracranial angioplasty or stent placement received aspirin and clopidogrel (Plavix) orally or via a nasogastric tube for at least 3 months after the procedure.

$\mathrm{BAO}$ was classified according to Archer and Horenstein ${ }^{16}$ into proximal (from the vertebrobasilar junction to the origin of the anterior inferior cerebellar artery), middle (from the origin of the anterior inferior cerebellar artery to the origin of the superior cerebellar artery), and distal occlusion (distal to the origin of the superior cerebellar artery). Revascularization status was assessed on the final angiogram and was classified according to the modified TICI scale ${ }^{17}$; successful revascularization was defined as modified TICI grades $2 \mathrm{~b}$ or 3 . All patients underwent nonenhanced CT immediately after and 24 hours after endovascular therapy for detection of intracranial hemorrhage.

\section{Outcome Measures}

For all patients, we assessed posterior circulation ASPECTS on pretreatment DWI, according to Tei et al. ${ }^{18}$ The presence or absence of thalamic infarction and its involved territory was also evaluated. The territory of thalamic infarctions was classified into anterior, posteromedial, ventrolateral, and posterolateral according to their arterial supply. ${ }^{19}$ Classification of BAO, revascularization status, posttreatment CT findings, posterior circulation ASPECTS, and thalamic infarction on pretreatment DWI were assessed by 2 neuroradiologists (with 3 and 5 years of experience, respectively) who were blinded to any clinical information. Conclusions were reached by consensus.

Neurologic evaluation was performed by a stroke neurologist immediately and 24 hours and 3 months after treatment; when any change occurred in clinical symptoms; and before the patient was discharged. Clinical outcome was assessed with the mRS by a stroke neurologist 3 months after treatment. When patients could not visit for neurologic evaluation, they were assessed via phone call. Good clinical outcome was defined as an mRS score of $\leq 2$. Symptomatic hemorrhage was defined as a documented hemorrhage associated with a decline of $\geq 4$ points in the NIHSS score or 1 -point deterioration in the level of consciousness.

\section{Statistical Analysis}

Sixty-two patients were divided into 2 groups for comparison, namely the ICAS group and the non-ICAS group. The baseline characteristics and clinical outcomes of the 2 groups were compared. Statistical analyses were performed with the use of SPSS software (Version 21.0; IBM, Armonk, New York). The $\chi^{2}$ test or Fisher exact test was used for categoric and binary variables, and the Mann-Whitney $U$ test, for continuous variables. $P<.05$ was considered significant.

\section{RESULTS}

Baseline patient characteristics are shown in Table 1. The mean age was 69 years, and $36(58.1 \%)$ were men. The etiology of ischemic stroke was cardioembolism in 32 patients (51.6\%), large- 
Table 1: Baseline characteristics of the study population

\begin{tabular}{lcccr}
\hline & $\begin{array}{c}\text { All Patients } \\
(\boldsymbol{n}=62)\end{array}$ & $\begin{array}{c}\text { Patients with } \\
\text { ICAS }(\boldsymbol{n}=15)\end{array}$ & $\begin{array}{c}\text { Patients without } \\
\text { ICAS }(\boldsymbol{n}=\mathbf{4 7})\end{array}$ & $\begin{array}{c}\boldsymbol{P} \\
\text { Value }\end{array}$ \\
\hline Age (mean) (yr) & $69.6 \pm 11.95$ & $67.1 \pm 10.03$ & $70.3 \pm 12.50$ & .205 \\
Male sex (No.) (\%) & $36(58.1)$ & $12(80.0)$ & $24(51.1)$ & .048 \\
Risk factor (No.) (\%) & & & & \\
$\quad$ Hypertension & $37(59.7)$ & $11(73.3)$ & $26(55.3)$ & .216 \\
$\quad$ Diabetes mellitus & $14(22.6)$ & $4(26.7)$ & $10(21.3)$ & .727 \\
Dyslipidemia & $16(25.8)$ & $6(40.0)$ & $10(21.3)$ & .182 \\
Smoking & $15(24.2)$ & $5(33.3)$ & $10(21.3)$ & .489 \\
$\quad$ Coronary artery disease & $5(8.1)$ & $0(0.0)$ & $5(10.6)$ & .323 \\
IV thrombolysis (No.) (\%) & $17(27.4)$ & $7(46.7)$ & $10(21.3)$ & .094 \\
Occlusion sites (No.) (\%) & & & & \\
$\quad$ Proximal & $12(19.4)$ & $9(60.0)$ & $3(6.4)$ & $<.001$ \\
$\quad$ Middle & $3(4.8)$ & $2(13.3)$ & $1(2.1)$ & .143 \\
$\quad$ Distal & $47(75.8)$ & $4(26.7)$ & $43(91.5)$ & $<.001$ \\
Bilateral thalamic infarction (No.) (\%) & $13(21.0)$ & $0(0.0)$ & $13(27.7)$ & .027 \\
Baseline NIHSS score (mean) & $12.4 \pm 6.23$ & $12.7 \pm 5.95$ & $12.3 \pm 6.37$ & .773 \\
pc-ASPECTS (median) (IQR) & $7(6-8)$ & $7(6-8)$ & $7(6-8)$ & .794 \\
Time to procedure (mean) (min) & $289.6 \pm 129.68$ & $309.3 \pm 139.65$ & $283.3 \pm 127.60$ & .532 \\
Procedure time (mean) (min) & $30.9 \pm 17.76$ & $39.0 \pm 19.47$ & $28.3 \pm 16.56$ & .006 \\
Time to revascularization (mean) (min) & $321.0 \pm 136.22$ & $348.3 \pm 143.84$ & $312.2 \pm 134.12$ & .459 \\
\hline
\end{tabular}

Note:-IQR indicates interquartile range; PC-ASPECTS, posterior circulation ASPECTS.
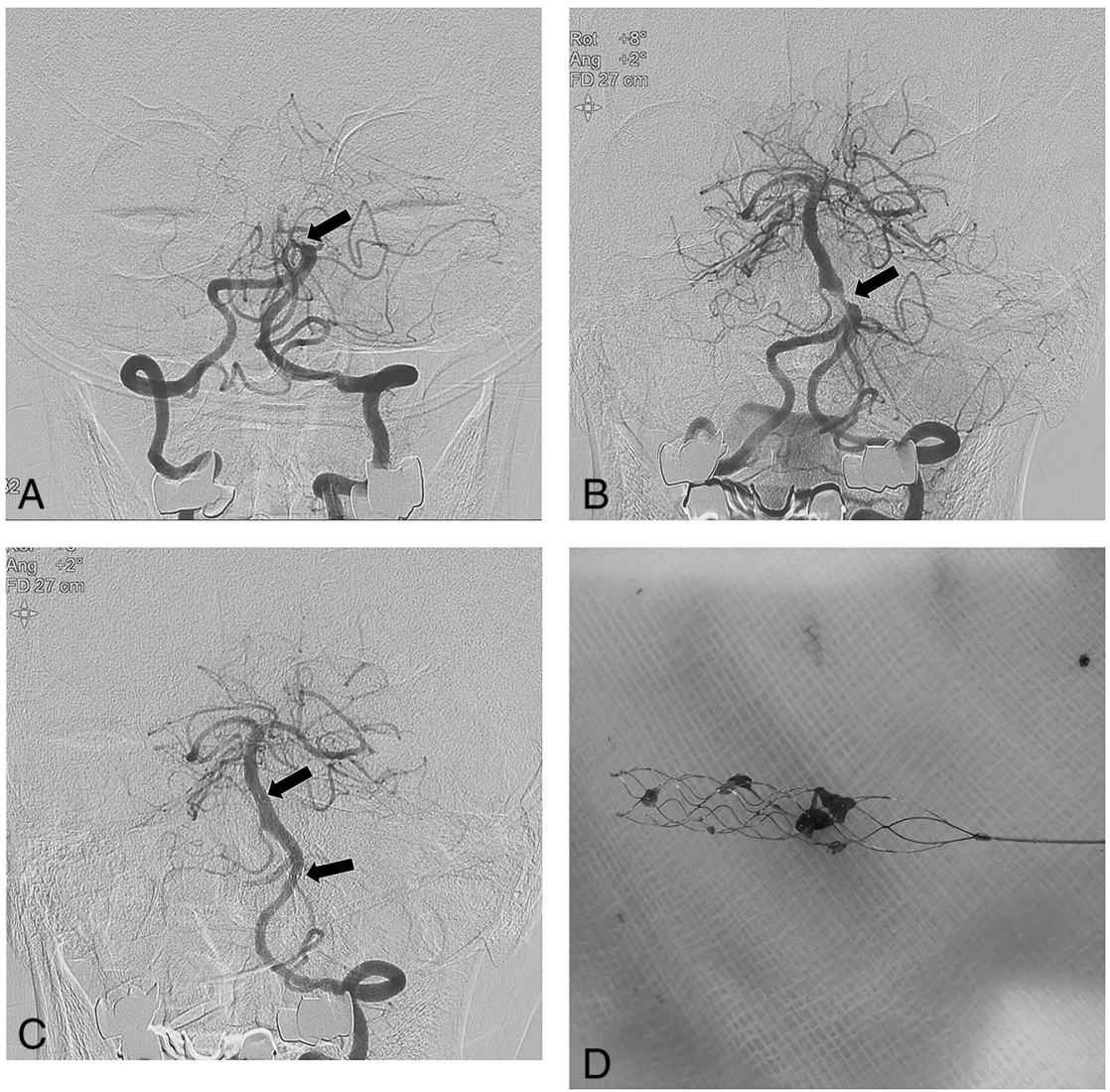

FIG 1. A 74-year-old male patient with acute stroke due to acute basilar artery occlusion. A, Left vertebral artery angiogram shows an occlusion (arrow) at the proximal segment of the basilar artery. $B$, Angiogrm obtained after 1 passage of the stent retriever reveals a severe underlying atherosclerotic stenosis (arrow) at the proximal segment of the basilar artery. C, Left vertebral angiogram obtained after intracranial angioplasty and stent placement shows complete recanalization of the basilar artery with good distal perfusion. Arrows indicate the proximal and distal ends of the Wingspan stent. $D$, Photograph shows small fragmented thrombi retrieved with a Solitaire stent.

artery atherosclerosis in 21 patients (33.9\%), and undetermined etiology in 9 patients (14.5\%). IV rtPA was administrated in 17 patients $(27.4 \%)$. The mean NIHSS score on admission was 12 , with scores ranging from 4 to 25 .
Of the 62 patients, $15(24.2 \%)$ had underlying ICAS at the occlusion site. Of these 15 patients, 1 had preocclusive severe stenosis on diagnostic angiography and 14 had occlusion on initial diagnostic angiography. These 14 patients had stenosis of $\geq 70 \%$ on follow-up angiography after first-line stent-retriever thrombectomy. Of 15 patients with underlying ICAS, 5 underwent intracranial angioplasty alone and 8 received angioplasty with stent placement (Fig 1). Two patients did not undergo angioplasty or stent placement due to device inaccessibility. Overall, $17.7 \%(11 / 62)$ of patients were treated by additional manual aspiration thrombectomy after failure of first-line stent-retriever thrombectomy.

The mean procedural time was longer in the ICAS group than in the non-ICAS group (39 minutes versus 28 minutes, $P=.006$ ). Occlusion in the proximal segment of the basilar artery was more frequent in the ICAS group $(9 / 15,60 \%)$ than in the non-ICAS group $(3 / 47,6.4 \%)(P<.001)$. In contrast, occlusion in the distal segment of the basilar artery was more frequent in the nonICAS group $(43 / 47,91.5 \%)$ than in the ICAS group $(4 / 15,26.7 \%)(P<.001)$. Otherwise, there were no significant differences between the 2 groups in age, sex, stroke risk factors, intravenous thrombolysis, time to procedure, time to revascularization, baseline NIHSS score, and DWI posterior circulation ASPECTS.

On a pretreatment DWI, thalamic infarction was seen in 24 patients (38.7\%). Of the 24 patients with thalamic infarctions, 13 had bilateral thalamic infarctions and 11 had unilateral thalamic infarctions. All bilateral thalamic infarctions occurred in paramedian regions. Five patients in the ICAS group had thalamic infarctions, and all of these 5 patients showed unilateral thalamic infarction. Nineteen patients in the non-ICAS group had thalamic infarction. Of these 19 patients, 13 had bilateral thalamic infarctions and 6 had unilateral thalamic infarction. There was no significant difference in the rate of thalamic infarction between the 2 groups, but the rate of bilateral thalamic infarction was significantly lower in the ICAS group $(0 / 15,0 \%)$ compared with the non-ICAS group $(13 / 47,27.7 \%)(P=.027)$. 
Table 2: Outcomes after endovascular therapy in 62 patients with acute stroke due to BAO

\begin{tabular}{lcccc}
\hline & $\begin{array}{c}\text { All Patients } \\
(\boldsymbol{n}=62)\end{array}$ & $\begin{array}{c}\text { Patients with } \\
\text { ICAS }(\boldsymbol{n}=15)\end{array}$ & $\begin{array}{c}\text { Patients without } \\
\text { ICAS }(\boldsymbol{n}=\mathbf{4 7})\end{array}$ & $\begin{array}{c}\boldsymbol{P} \\
\text { Value }\end{array}$ \\
\hline mTICl 2b or 3 (No.) (\%) & $60(96.8)$ & $15(100.0)$ & $45(95.7)$ & NS \\
mRS 0-2 (No.) (\%) & $33(53.2)$ & $9(60.0)$ & $24(51.1)$ & NS \\
Symptomatic hemorrhage (No.) (\%) & $1(1.6)$ & $0(0.0)$ & $1(2.1)$ & NS \\
Mortality (No.) (\%) & $10(16.1)$ & $2(13.3)$ & $8(17.0)$ & NS \\
\hline
\end{tabular}

Note:- mTICl indicates modified TICl; NS, nonsignificant.

The clinical outcomes of 62 patients are summarized in Table 2. Overall, successful revascularization (modified TICI grades $2 \mathrm{~b}$ or 3) was achieved in $96.8 \%(60 / 62)$ of the patients, and favorable outcomes (mRS $0-2)$, in $53.2 \%$ (33/62) of the patients. There was 1 patient $(1.6 \%)$ with symptomatic hemorrhage, and the mortality rate was $16.1 \%(10 / 62)$. There was no significant difference between the 2 groups in the rates of successful revascularization, favorable outcome, symptomatic hemorrhage, and mortality.

\section{DISCUSSION}

The main findings of our study are as follows: 1) Underlying ICAS of $\geq 70 \%$ was identified in $24.2 \%$ of the patients with acute ischemic stroke due to BAO; 2) occlusion in the proximal segment was more frequently seen in patients with $\mathrm{BAO}$ with underlying ICAS, whereas occlusion in the distal segment was more frequently observed in those without underlying ICAS; and 3) bilateral thalamic infarction on pretreatment DWI was seen only in patients without underlying ICAS.

The incidence of underlying ICAS in acute ischemic stroke caused by intracranial large-artery occlusion has been reported to be $17 \%-60 \% .^{10,11,14}$ It is well-known that ICAS is more prevalent in Asian countries, and accordingly, the highest incidence was reported by a Chinese center. ${ }^{10}$ The results of our study are in line with those reports; $24 \%$ of patients with acute BAO had underlying ICAS of $\geq 70 \%$. The possibility of underlying ICAS should be considered when performing endovascular therapy in patients with acute BAO, especially when encountering a difficulty during mechanical thrombectomy in regions where ICAS is more prevalent. The primary revascularization rate of stent-retriever thrombectomy in patients with acute stroke due to large-vessel occlusions was reported to be up to $70 \%-80 \% .{ }^{13}$ Underlying ICAS in the occluded artery can be an important cause of failed stentretriever thrombectomy, especially in Asian patients. ${ }^{13,14}$

Predicting the presence of underlying ICAS before and during stent-retriever thrombectomy will be helpful for neurointerventionists for appropriate therapeutic planning. Our study suggested that the occlusion site can be a useful marker in predicting underlying ICAS in patients with acute BAO. In our study, 73\% of patients with underlying ICAS had occlusions in the proximal and middle segments of the basilar artery, while $92 \%$ of those without underlying ICAS had occlusions in distal segment of the basilar artery. The results from our study are in accordance with those in a recently published study by Kim et al, ${ }^{11}$ which included 51 patients with acute vertebrobasilar artery occlusion. They found that $69.3 \%$ of patients with ICAS had occlusions in the proximal and middle segments of the basilar artery, while $87.5 \%$ of those with embolism had occlusions in the distal segment.

Thalamic infarctions are often detected on a pretreatment DWI in patients with acute BAO. ${ }^{7}$ However, to our knowledge, there have been no reports regarding the association between thalamic infarction on a pretreatment DWI and underlying ICAS. In our study, there was no significant difference between the ICAS group and the non-ICAS group in the incidence of thalamic infarction; however, the incidence of bilateral thalamic infarctions differed significantly between patients with underlying ICAS and those without it. No patients with underlying ICAS showed bilateral thalamic infarctions on pretreatment DWI. This difference might be related to the occlusion segment of the basilar artery. In this study, all bilateral thalamic infarction occurred in the bilateral paramedian region, which is supplied by a single thalamoperforating artery (the artery of Percheron). ${ }^{20,21}$ The artery of Percheron arises from the proximal P1 segment of the posterior cerebral artery. In case of occlusion in the distal basilar artery, the emboli may easily extend to the P1 segment of the posterior cerebral artery, which would result in occlusion of the artery of Percheron and cause bilateral paramedian thalamic infarction. In contrast, patients with ICAS are not likely to have paramedian thalamic infarctions because occlusions of the proximal and middle segment are more common and the P1 segments are usually preserved in patients with ICAS. Our study suggested that if bilateral paramedian thalamic infarctions are seen on pretreatment DWI in patients with BAO, the possibility of the presence of underlying ICAS would be low.

The optimal treatment for patients with acute BAO with underlying ICAS remains unclear. In the present study, $86.7 \%$ of patients (13/15) with underlying ICAS were treated with intracranial angioplasty with or without stent placement after first-line mechanical thrombectomy. We achieved high rates of successful revascularization (100\%) and favorable outcome (60\%), no symptomatic hemorrhage, and a low mortality rate $(13.3 \%)$ in these 15 patients. Recently, Yoon et $\mathrm{al}^{14}$ reported the results of emergent angioplasty and stent placement in 40 patients with acute ischemic stroke due to underlying ICAS. They reported high rates of successful revascularization (95\%) and favorable outcome $(62.5 \%)$ and low rates of symptomatic hemorrhage (7.5\%) and mortality (15\%). They suggested that intracranial angioplasty with or without stent placement is safe and feasible in hyperacute stroke secondary to underlying ICAS. Gao et $\mathrm{al}^{10}$ also reported a $100 \%$ successful revascularization rate and a favorable 3-month outcome rate of $46 \%$ in a series of 13 patients with acute $\mathrm{BAO}$ and underlying ICAS who were treated with stent-retriever thrombectomy and intracranial stent placement.

In contrast to our study, Kim et $\mathrm{al}^{11}$ recently reported a low rate of good outcome (10.5\% with mRS $0-2$ at 3 months) and a high mortality rate $(21.1 \%)$ after endovascular therapy in 19 patients with acute vertebrobasilar occlusion due to ICAS. The main reasons for the poor outcomes after endovascular therapy in the study of Kim et al may be heterogeneity of the rescue treatment after primary mechanical thrombectomy, the low rate of angioplasty/stent placement as a rescue treatment, and long procedural time. In their study, one-third of patients received intra-arterial infusion of tirofiban, one-third of patients underwent angioplasty alone, and another one-third of patients underwent stent place-

AJNR Am J Neuroradiol 38:1600-04 Aug 2017 www.ajnr.org

1603 
ment. The results of our study together with previous reports suggest that intracranial angioplasty with or without stent placement is promising in patients with acute BAO due to underlying ICAS. In addition, the procedural time was much longer in the study of Kim et al ( 96 versus 39 minutes in patients with ICAS and 61 versus 28 minutes in those without ICAS) than in our study.

Our study had several limitations, including a retrospective, noncontrolled study design and a limited number of patients. Prospective, multicenter studies are needed to further determine the clinical and radiologic characteristics of patients with BAO with underlying ICAS. In addition, prospective clinical trials are also needed to confirm the efficacy and safety of intracranial angioplasty and stent placement in patients with $\mathrm{BAO}$ with underlying ICAS compared with other treatment options such as intraarterial infusion of antiplatelet drugs.

\section{CONCLUSIONS}

Underlying ICAS is common in patients with acute stroke due to BAO. The occlusion site can be used as a useful marker for predicting underlying ICAS in patients with acute BAO. There was no difference in treatment outcomes after endovascular therapy between patients with underlying ICAS and those without it.

\section{REFERENCES}

1. Schonewille WJ, Wijman CA, Michel P, et al; BASICS study group. Treatment and outcomes of acute basilar artery occlusion in the Basilar Artery International Cooperation Study (BASICS): a prospective registry study. Lancet Neurol 2009;8:724-30 CrossRef Medline

2. Mortimer AM, Bradley M, Renowden SA. Endovascular therapy for acute basilar artery occlusion: a review of the literature. J Neurointerv Surg 2012;4:266-73 CrossRef Medline

3. Yeung JT, Matouk CC, Bulsara KR, et al. Endovascular revascularization for basilar artery occlusion. Interv Neurol 2015;3:31-40 CrossRef Medline

4. Gory B, Eldesouky I, Sivan-Hoffmann R, et al. Outcomes of stent retriever thrombectomy in basilar artery occlusion: an observational study and systematic review. J Neurol Neurosurg Psychiatry 2016;87:520-25 CrossRef Medline

5. Möhlenbruch M, Stampfl S, Behrens L, et al. Mechanical thrombectomy with stent retrievers in acute basilar artery occlusion. $A J N R$ Am J Neuroradiol 2014;35:959-64 CrossRef Medline

6. Baek JM, Yoon W, Kim SK, et al. Acute basilar artery occlusion: outcome of mechanical thrombectomy with Solitaire stent within $\mathbf{8}$ hours of stroke onset. AJNR Am J Neuroradiol 2014;35:989-93 CrossRef Medline
7. Yoon W, Kim SK, Heo TW, et al. Predictors of good outcome after stent-retriever thrombectomy in acute basilar artery occlusion. Stroke 2015;46:2972-75 CrossRef Medline

8. Mattle HP, Arnold M, Lindsberg PJ, et al. Basilar artery occlusion. Lancet Neurol 2011;10:1002-14 CrossRef Medline

9. Bang OY. Intracranial atherosclerosis: current understanding and perspectives. J Stroke 2014;16:27-35 CrossRef Medline

10. Gao F, Lo WT, Sun X, et al. Combined use of mechanical thrombectomy with angioplasty and stenting for acute basilar occlusions with underlying severe intracranial vertebrobasilar stenosis: preliminary experience from a single Chinese center. AJNR Am J Neuroradiol 2015;36:1947-52 CrossRef Medline

11. Kim YW, Hong JM, Park DG, et al. Effect of intracranial atherosclerotic disease on endovascular treatment for patients with acute vertebrobasilar occlusion. AJNR Am J Neuroradiol 2016;37:2072-78 CrossRef Medline

12. Adams HP Jr, Bendixen BH, Kappelle LJ, et al. Classification of subtype of acute ischemic stroke. Definitions for use in a multicenter clinical trial. TOAST. Trial of Org 10172 in Acute Stroke Treatment. Stroke 1993;24:35-41 Medline

13. Kim SK, Yoon W, Moon SM, et al. Outcomes of manual aspiration thrombectomy for acute ischemic stroke refractory to stent-based thrombectomy. J Neurointerv Surg 2015;7:473-77 CrossRef Medline

14. Yoon W, Kim SK, Park MS, et al. Endovascular treatment and the outcomes of atherosclerotic intracranial stenosis in patients with hyperacute stroke. Neurosurgery 2015;76:680-86 discussion 686 CrossRef Medline

15. Samuels OB, Joseph GJ, Lynn MJ, et al. A standardized method for measuring intracranial arterial stenosis. AJNR Am J Neuroradiol 2000;21:643-46 Medline

16. Archer CR, Horenstein S. Basilar artery occlusion: clinical and radiological correlation. Stroke 1977;8:383-90 CrossRef Medline

17. Zaidat OO, Yoo AJ, Khatri P, et al; STIR Revascularization working group, STIR Thrombolysis in Cerebral Infarction (TICI) Task Force. Recommendations on angiographic revascularization grading standards for acute ischemic stroke: a consensus statement. Stroke 2013;44:2650-63 CrossRef Medline

18. Tei H, Uchiyama S, Usui T, et al. Posterior circulation ASPECTS on diffusion-weighted MRI can be a powerful marker for predicting functional outcome. J Neurol 2010;257:767-73 CrossRef Medline

19. Song YM. Topographic patterns of thalamic infarcts in association with stroke syndromes and aetiologies. J Neurol Neurosurg Psychiatry 2011;82:1083-86 CrossRef Medline

20. Lazzaro NA, Wright B, Castillo M, et al. Artery of percheron infarction: imaging patterns and clinical spectrum. AJNR Am JNeuroradiol 2010;31:1283-89 CrossRef Medline

21. Li X, Agarwal N, Hansberry DR, et al. Contemporary therapeutic strategies for occlusion of the artery of Percheron: a review of the literature. J Neurointerv Surg 2015;7:95-98 CrossRef Medline 